\title{
Regulating effect of MMP-9 and TIMP-1 in pi- tuitary adenoma invasion
}

\author{
J.H. Mao', H. Guo', N. Si², L. Qiü, L.F. Guo ${ }^{4}$, Z.S. Sun', Y. Xiang', X.H. Yang', \\ W.G. Zhao ${ }^{1}$ and W.C. Zhang ${ }^{1}$ \\ 1Department of Neurosurgery, Harrison International Peace Hospital attached to \\ Hebei Medical University, Hengshui, Hebei, China \\ ${ }^{2}$ Department of Anesthesiology, Harrison International Peace Hospital attached to \\ Hebei Medical University, Hengshui, Hebei, China \\ ${ }^{3}$ Department of Pathology, Harrison International Peace Hospital attached to Hebei \\ Medical University, Hengshui, Hebei, China \\ ${ }^{4}$ Department of Laboratory Medicine, Harrison International Peace Hospital \\ attached to Hebei Medical University, Hengshui, Hebei, China \\ Corresponding author: Z.S. Sun \\ E-mail: zhaoshenghs3sun@163.com
}

Genet. Mol. Res. 14 (4): 17091-17098 (2015)

Received August 13, 2015

Accepted October 11, 2015

Published December 16, 2015

DOI http://dx.doi.org/10.4238/2015.December.16.9

\begin{abstract}
Pituitary adenomas can cause endocrine disorder and organ damage, with some aggressive ones leading to a high postoperative recurrence rate. The occurrence and development of these type of tumors is closely related with matrix metalloproteinases (MMPs) and endogenous specific tissue inhibitor of MMPs (TIMPs). In this study, the relationship between pituitary adenoma invasion and the changes in MMP-8 and TIMP1 expressions is analyzed. Specimens from sixty patients with pituitary adenoma were collected in our hospital after surgery, including thirty cases of invasive pituitary adenomas and thirty cases of noninvasive pituitary adenomas. Western blotting and real-time PCR were used to detect MMP8/TIMP-1 protein and mRNA levels, respectively, in the two types of pituitary adenomas, while ELISA was used to detect both compounds' levels in the patient's serum. Compared with noninvasive pituitary adenomas, MMP-
\end{abstract}


8 was significantly overexpressed in invasive pituitary adenomas, while TIMP-1 was obviously lower ( $P<0.05$ for both). Moreover, MMP-8 mRNA expression in invasive pituitary adenomas was significantly higher than in noninvasive pituitary adenomas, while TIMP-1 mRNA expression was markedly lower ( $P<0.05$ for both). Finally, MMP-8 expression in the serum is upregulated in patients with invasive pituitary adenomas relative to the noninvasive ones, and the expression of TIMP-1 significantly reduced ( $P<$ 0.05 for both). These results show that increased MMP-8 and decreased TIMP-1 expressions are closely related to the invasive pituitary adenoma, and can be helpful for the evaluation.

Key words: Pituitary adenoma; MMP-8; TIMP-1; Invasion

\section{INTRODUCTION}

Pituitary adenomas are mostly benign intracranial tumors that have high incidence, just below the incidence of gliomas and meningiomas. In recent years, their incidence has been increasing, particularly in younger age groups (Manara et al., 2014; Oklu et al., 2014). Pituitary adenoma can stimulate excess hormone secretion in the pituitary gland and cause an endocrine disease, which can affect the whole body and cause tissue damage. Tumor-related hypopituitarism brings pain to the patient, seriously affects the quality of survival, and can even lead to death (Babbo et al., 2014). Some pituitary adenomas have been proven invasive, being able to invade the cavernous sinus, sphenoid sinus, saddle pool and saddle surrounding the brain tissue, leading to damage on brain tissue, the vomer surrounding area, the internal carotid artery, and the cranial nerve. Invasive pituitary adenoma can possess malignant tumor characteristics that can lead to low complete excision rates. Even after receiving post-surgery auxiliary radiotherapy or chemotherapy, its recurrence is still high. It is extremely difficult to cure and it has become an important human health problem (Ding et al., 2014; Kwancharoen et al., 2014). The pathogenesis of invasive pituitary adenoma is a complicated process involving many factors and multiple steps (Broder et al., 2014). At present, it is difficult to find an objective evaluation method for invasive pituitary adenoma, with the only viable option being a combination of clinical symptoms, imaging and pathological histology (Yedinak and Fleseriu, 2014). Finding ways to determine invasive pituitary adenoma in the laboratory may provide a theoretical reference for the clinical diagnosis of the type of pituitary adenoma, which has an important significance for clinical application.

Matrix metalloproteinases (MMPs) are a family of calcium/zinc-dependent endogenous proteases that can degrade all kinds of extracellular matrix (ECM) proteins. They play a key role in maintaining the process of dynamic and physiologic balance of the ECM (Mavrogonatou et al., 2014; Verma et al., 2014). MMPs can be inhibited by endogenous specific tissue inhibitors of metalloproteinases (TIMPs; Bottino et al., 2014), which maintain the integrity of the ECM and basement membrane together. The dynamic balance between MMPs and TIMPs is altered during the process of tumor occurrence and infiltration, leading to the destruction of the ECM and cell basement membrane integrity. This results in the increase of tumor angiogenesis, further promoting tumor invasion and metastasis (Lee et al., 2014; Ma et al., 2014). However, whether the expression of MMP-8 and TIMP-1 in pituitary adenomas and their dynamic balance can be used as markers for pituitary adenoma has not been elucidated. This study aims to explore the influence of MMP-8 and TIMP-1 on pituitary adenoma invasion by detecting their expression changes. 


\section{MATERIAL AND METHODS}

\section{Subjects}

Specimens from sixty patients with pituitary adenoma were collected in our hospital between January 2013 and December 2014. The enrolled subjects group had a mean age of 32.1 \pm 17.25 (23-57) years, and was composed of 32 males and 28 females. All patients received surgery, including 30 cases of invasive pituitary adenomas and 30 cases of noninvasive pituitary adenomas. All clinical symptoms were consistent with the diagnostic criteria for pituitary adenoma, and confirmed by clinical imaging, surgery, and pathology diagnosis. Patients did not use growth hormone inhibitor drugs preoperatively. The general clinical information showed no statistical differences $(P>0.05)$. Subjects signed informed consents before the study and the study was approved by the ethics committee in our hospital. The specimens were preserved in liquid nitrogen under aseptic conditions.

\section{Reagents}

PVDF transfer membranes were bought from Pall Life Sciences (Port Washington, NY, USA). EDTA was purchased from GE Healthcare (Logan, UT, USA). Western blot-related chemical reagents were purchased from Shanghai Beyotime Biotechnology Co., Ltd. The ECL reagent was bought from GE Healthcare. The TRIzol reagent, RNA extraction kit, RT-PCR primers, reverse transcription (RT) kit, and real-time PCR reagents were bought from Invitrogen, USA. The CIRP primary antibody was purchased from DPC Biermann $\mathrm{GmbH}$. The horseradish peroxidase (HRP)-conjugated IgG secondary antibody was bought from Cell Signaling Technology, USA. $\beta$-actin was purchased from Santa Cruz Biotechnology, USA. The ELISA kit was bought from R\&D Systems, USA.

\section{Western blotting}

The tissue was digested using a lysis buffer. Proteins were separated using a $10 \%$ SDSPAGE. Quantification was performed through chemiluminescence using Quantity One (Bio-Rad Laboratories, Inc., USA). Antibody dilutions for $\beta$-catenin, MMP-8, and TIMP-1 were 1:2000, 1:500, and 1:500, respectively. Protein levels were normalized to $\beta$-actin and the experiments were performed in quadruplicates.

\section{Real-time PCR}

The cDNA was synthesized with $1 \mu \mathrm{g}$ RNA from the samples. The primers used are as follows. MMP-8 forward primer: 5'-CTACGGAAGATCTCAGCGAAT-3', reverse primer: 5'-CTCTGGGACATCTCCGTCA-3', TIMP forward primer: 5'-GGAAGATCCTACTCAATAGCG-3', reverse primer: 5'-GGGACTCTCATCGTCATCC-3'; $\beta$-actin: forward primer: 5'-AGTCTAGTTGCTG G-GTACC-3', reverse primer: 5'-TAATAGGATGTCTGGACTCCG-3'. The cycling conditions consisted of an initial, single cycle of $1 \mathrm{~min}$ at $52^{\circ} \mathrm{C}$, followed by 35 cycles of $30 \mathrm{~s}$ at $90^{\circ} \mathrm{C}, 50 \mathrm{~s}$ at $58^{\circ} \mathrm{C}$, and $35 \mathrm{~s}$ at $72^{\circ} \mathrm{C}$. PCR amplifications were performed in triplicates for each sample. Gene expression levels were quantified relative to the expression of GAPDH using an optimized comparative Ct method $\left({ }^{\Delta} \mathrm{Ct}\right)$. 


\section{ELISA}

The peripheral blood was acquired before the surgery. After being centrifuged at $2000 \mathrm{rpm}$ for $15 \mathrm{~min}$, the serum was collected and stored at $-80^{\circ} \mathrm{C}$. ELISA kits were used to detect MMP-8 and TIMP expression changes according to the manual. The diluted standard product $(50 \mu \mathrm{L})$ was put into the corresponding reaction holes to prepare the standard curve, after which the samples $(50 \mu \mathrm{L})$ were added to each hole. After washing the plate for five times, $50 \mu$ l enzyme reagent was added to each hole. After incubated at $37^{\circ} \mathrm{C}$ for $30 \mathrm{~min}$, the plate was washed again for five times. Agent $A(50 \mu \mathrm{L})$ and agent $B(50 \mu \mathrm{L})$ were added to each hole and the plate was incubated at $37^{\circ} \mathrm{C}$ for $10 \mathrm{~min}$. The reaction was terminated by adding $50 \mu \mathrm{l}$ stop solution. The plate was measured at $450 \mathrm{~nm}$ to obtain the OD value. The sample concentration was calculated from the OD value and standard curve.

\section{Statistical analysis}

Numerical data are reported as means \pm SD. Differences between multiple groups were analyzed using one-ANOVA. All statistical analyses were performed using the SPSS software (version 13.0; Chicago, IL, USA). $P<0.05$ was considered as a significant difference.

\section{RESULTS}

\section{General information}

The general information collected from the invasive and noninvasive pituitary adenoma patients was compared. Invasive pituitary adenoma patients exhibited a younger onset age of 32.1 \pm 17.25 years. The two groups presented similar gender incidence with no statistical difference $(P$ $>0.05$; Table 1).

Table 1. Clinical information of patients and their pituitary adenomas.

\begin{tabular}{lccr}
\hline Type of pituitary adenoma $(\mathrm{N})$ & Gender $(\mathrm{M} / \mathrm{F})$ & Age $($ years $)$ & Tumor volume $\left(\mathrm{mm}^{3}\right)$ \\
\hline Noninvasive $(20)$ & $17 / 13$ & $34.2 \pm 12.65$ & $20.1 \pm 5.31$ \\
Invasive $(20)$ & $14 / 16$ & $32.1 \pm 14.36$ & $21.6 \pm 6.16$ \\
\hline
\end{tabular}

\section{Changes in MMP-8 and TIMP-1 protein expression in pituitary adenomas}

Western blotting was used to analyze the protein expression changes of MMP-8 and TIMP-1 between invasive and noninvasive pituitary adenomas. MMP-8 protein expression in noninvasive pituitary adenomas was lower when compared with the invasive pituitary adenoma $(P<$ 0.05; Figure 1C). Compared with the noninvasive pituitary adenoma, TIMP-1 protein expression was lower in invasive pituitary adenoma $(P<0.05$; Figure $1 C)$. These indicate that overexpressed MMP-8 may promote pituitary adenoma invasion, while a reduction of TIMP-1 may decline the inhibitory effect on MMPs, and thus further promoting tumor occurrence and development. 


\section{Changes on MMP-8 and TIMP-1 mRNA expressions in pituitary adenomas}

Real-time PCR was used to analyze changes on the MMP-8 and TIMP-1 mRNA expressions between invasive and noninvasive pituitary adenomas. MMP-8 mRNA expression increased significantly when the pituitary adenoma is invasive $(P<0.05$; Figure 2$)$. Compared with the noninvasive pituitary adenoma, TIMP-1 mRNA expression was lower in the invasive pituitary adenoma $(P<0.05$; Figure 2). These suggest that MMP-8 and TIMP-1 mRNA expressions have similar trends to their respective protein expressions.
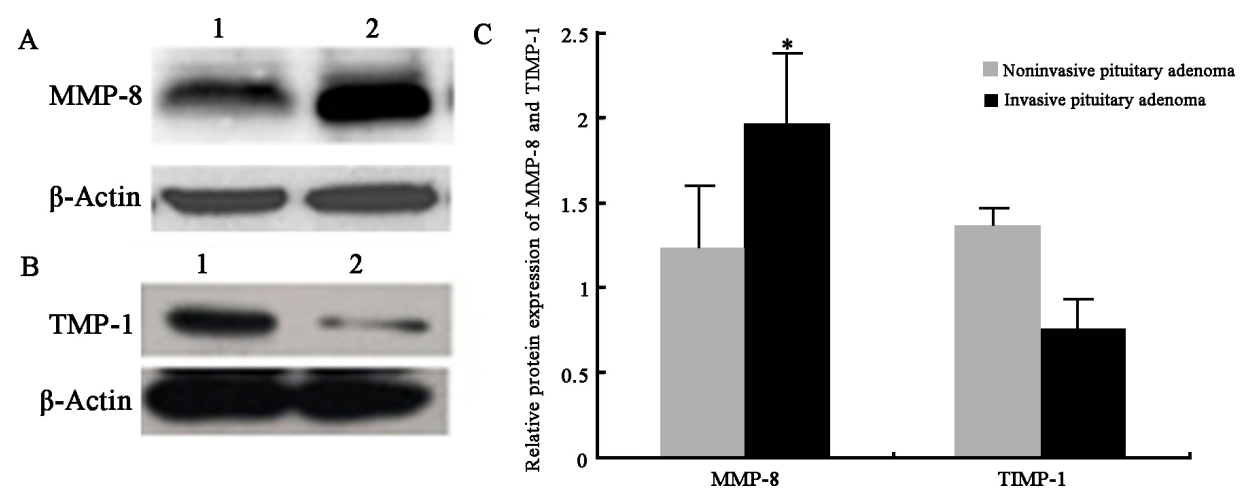

Figure 1. Protein expression changes of MMP-8 and TIMP-1 in pituitary adenomas. A. MMP-8 protein expression in pituitary adenomas: lane 1: noninvasive pituitary adenoma, lane 2: invasive pituitary adenoma; B. TIMP-1 protein expression in pituitary adenomas: lane 1: noninvasive pituitary adenoma, lane 2: invasive pituitary adenoma; C. Statistical analysis of MMP-8 and TIMP-1 protein expressions in pituitary adenomas. ${ }^{*} P<0.05$, compared with noninvasive pituitary adenoma.

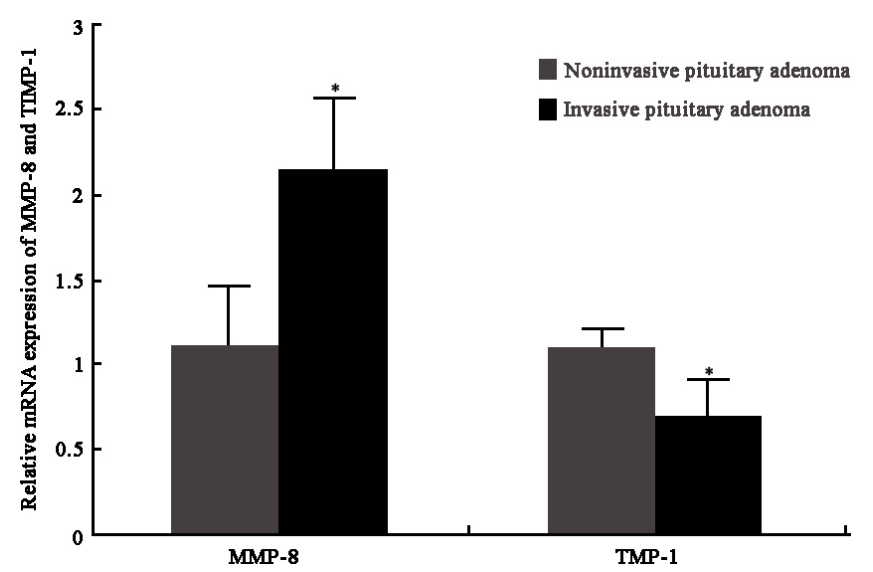

Figure 2. Statistical analysis of MMP-8 and TIMP-1 mRNA expressions in pituitary adenomas. ${ }^{*} \mathrm{P}<0.05$, compared with noninvasive pituitary adenoma.

\section{Changes on MMP-8 and TIMP-1 expressions in the serum of pituitary adenoma patients}

ELISA was performed on serum samples from patients with invasive and noninvasive 
pituitary adenomas to analyze the changes on MMP-8 and TIMP-1 expressions. MMP-8 mRNA expression was higher in the invasive pituitary adenoma $(P<0.05$; Figure 3$)$. Compared with the noninvasive pituitary adenoma, TIMP-1 mRNA was lower in the invasive pituitary adenoma $(\mathrm{P}<$ 0.05 ; Figure 3). These reinforce the previous results on MMP-8 and TIMP-1 promoting the invasion by pituitary adenomas.

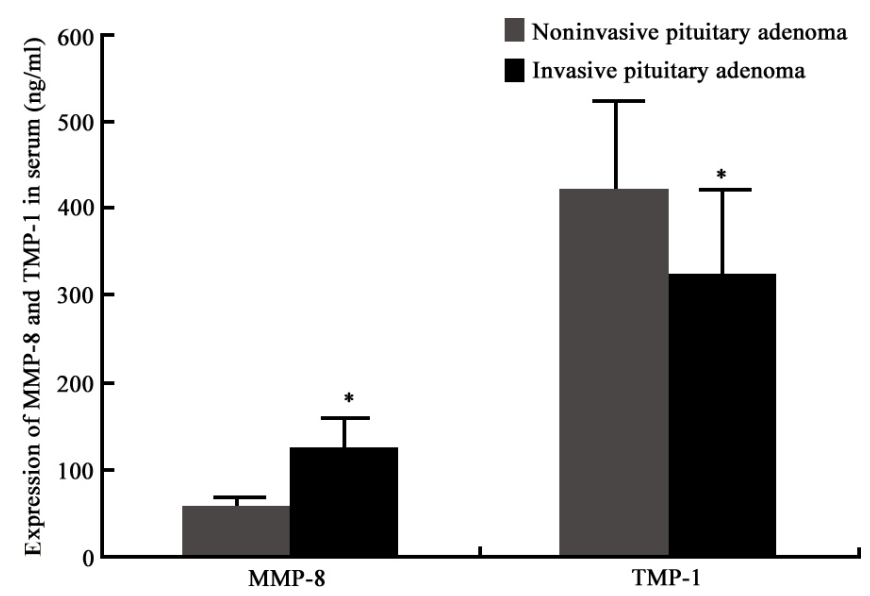

Figure 3. Statistical analysis of MMP-8 and TIMP-1 expressions in the serum of pituitary adenoma patients. ${ }^{*} P<0.05$, compared with noninvasive pituitary adenoma.

\section{DISCUSSION}

The invasion mechanism of pituitary adenoma is complex and interacts with the nervous, endocrine, and immune systems. A variety of factors are involved this process, which not only includes gene changes that affect the balance of cell proliferation and apoptosis, but also ECM changes (Babichenko et al., 2014; Ma et al., 2015).

MMPs are a family of zinc-dependent enzyme composed of at least 26 members. MMPs are usually secreted to the ECM in a latent form, called zymogen. They can only be activated through the removal of the pro-peptide domain, which can be achieved through other proteolytic enzymes or corresponding activating objects, and can combine or degrade ECM in a specific manner (Bi et al., 2015). Different MMPs can degrade the same kind of ECM components, and a MMP family can act on different ECM components. Previous studies confirmed that MMP-9 plays a role in pituitary adenoma invasion (Kim et al., 2014; Agren et al., 2015). However, the role of MMP-8, another important member of the MMPs family, in pituitary adenoma invasion has not been elucidated yet. TIMPs are endogenous specific MMPs inhibitors that are composed of multiple members (Ahmed Haji Omar et al., 2015). A study about the expression and balance of MMP-8 and TIMP-1 in the pituitary adenoma invasion is still lacking.

The selected pituitary adenoma cases in this study are mainly in a young age, and with similar incidence between men and women. The general information regarding age and gender showed no statistical differences, which is consistent with previous studies (Ozgören et al., 2014). This effect damages the physical barrier around tumor cells, increasing the tumor biological activity by degrading the matrix components, which allows the tumor to escape from the immune system. The changes of the ECM lead to an increase of tumor angiogenesis, leading to further pituitary 
adenoma proliferation and invasive growth (Ramanujum et al., 2013; Pereira et al., 2014).

To sum up, this study confirmed that the overexpressed MMP-8 and decreased TIMP-1 are closely related to the invasive feature of pituitary adenomas. They can be treated as biomarkers to assess pituitary adenoma invasion. Furthermore, reversing the abnormal expression of MMP-8 and TIMP-1 may help suppressing pituitary adenoma invasion.

\section{Conflicts of interest}

The authors declare no conflict of interest.

\section{ACKNOWLEDGMENTS} item (\#14003A).

Research supported by the Hebei Province Hengshui City Science and Technology Plan

\section{REFERENCES}

Agren MS, Schnabel R, Christensen LH and Mirastschijski U (2015). Tumor necrosis factor- $\alpha$-accelerated degradation of type I collagen in human skin is associated with elevated matrix metalloproteinase (MMP)-1 and MMP-3 ex vivo. Eur. J. Cell Biol. 94: 12-21.

Ahmed Haji Omar A, Haglund C, Virolainen S, Häyry V, et al. (2015). MMP-7, MMP-8, and MMP-9 in oral and cutaneous squamous cell carcinomas. Oral Surg., Oral Med., Oral Pathol., Oral Radiol. \& Endod. 119: 459-467.

Babbo A, Kalapurakal GT, Liu B, Bajramovic S, et al. (2014). The presence of a pituitary tumor in patients with prostate cancer is not a contraindication for leuprolide therapy. Int. Urol. Nephrol. 46: 1775-1778.

Babichenko II, Andriukhin MI, Pulbere S and Loktev A (2014). Immunohistochemical expression of matrix metalloproteinase-9 and inhibitor of matrix metalloproteinase-1 in prostate adenocarcinoma. Int. J. Clin. Exp. Pathol. 7: 9090-9098.

Bi HX, Shi HB, Zhang T and Cui G (2015). PRDM14 promotes the migration of human non-small cell lung cancer through extracellular matrix degradation in vitro. Chin. Med. J. (Beijing, China, Engl. Ed.) 128: 373-377.

Bottino J, Gelaleti GB, Maschio LB, Jardim-Perassi BV, et al. (2014). Immunoexpression of ROCK-1 and MMP-9 as prognostic markers in breast cancer. Acta Histochem. 116: 1367-1373.

Broder MS, Neary MP, Chang E, Cherepanov D, et al. (2014). Treatments, complications, and healthcare utilization associated with acromegaly: a study in two large United States databases. Pituitary 17: 333-341.

Ding D, Yen CP, Starke RM, Lee CC, et al. (2014). Unyielding progress: recent advances in the treatment of central nervous system neoplasms with radiosurgery and radiation therapy. J. Neurooncol. 119: 513-529.

Kim GE, Lee JS, Choi YD, Lee KH, et al. (2014). Expression of matrix metalloproteinases and their inhibitors in different immunohistochemical-based molecular subtypes of breast cancer. BMC Cancer 14: 959.

Kwancharoen R, Blitz AM, Tavares F, Caturegli P, et al. (2014). Clinical features of sellar and suprasellar meningiomas. Pituitary 17: 342-348.

Lee GR, Jang SH, Kim CJ, Kim AR, et al. (2014). Capsaicin suppresses the migration of cholangiocarcinoma cells by down-regulating matrix metalloproteinase-9 expression via the AMPK-NF-KB signaling pathway. Clin. Exp. Metastasis 31: 897-907.

Ma C, Wu B, Huang X, Yuan Z, et al. (2014). SUMO-specific protease 1 regulates pancreatic cancer cell proliferation and invasion by targeting MMP-9. Tumour Biol. 35: 12729-12735.

Ma J, Shi J, Zhao D, Cheng L, et al. (2015). Raf kinase inhibitor protein inhibits cholangiocarcinoma cell metastasis by downregulating matrix metalloproteinase 9 and upregulating tissue inhibitor of metalloproteinase 4 expression. Oncol. Lett. 9: 15-24.

Manara R, Gabrieli J, Citton V, Ceccato F, et al. (2014). Intracranial internal carotid artery changes in acromegaly: a quantitative magnetic resonance angiography study. Pituitary 17: 414-422.

Mavrogonatou E, Angelopoulou MT and Kletsas D (2014). The catabolic effect of TNFa on bovine nucleus pulposus intervertebral disc cells and the restraining role of glucosamine sulfate in the TNFa-mediated up-regulation of MMP-3. J. Orthop. Res. 32: 1701-1707.

Oklu R, Deipolyi AR, Wicky S, Ergul E, et al. (2014). Identification of small compound biomarkers of pituitary adenoma: a bilateral inferior petrosal sinus sampling study. J. Neurointerv. Surg. 6: 541-546. 
Ozgören O, Develioglu H, Güncü G, Akman A, et al. (2014). The adjunctive effect of tenoxicam during non-surgical periodontal treatment on clinical parameters and gingival crevicular fluid levels of MMP-8 and TNF- $\alpha$ in patients with chronic periodontitis - randomized, double-blind clinical trial. Adv. Clin. Exp. Med. 23: 559-565.

Pereira FV, Ferreira-Guimarães CA, Paschoalin T, Scutti JA, et al. (2014). A natural bacterial-derived product, the metalloprotease arazyme, inhibits metastatic murine melanoma by inducing MMP-8 cross-reactive antibodies. PLoS One 9: e96141.

Ramanujum R, Lin YL, Liu JK and He S (2013). Regulatory expression of MMP-8/MMP-9 and inhibition of proliferation, migration and invasion in human lung cancer A549 cells in the presence of HGF variants. Kaohsiung J. Med. Sci. 29: 530-539.

Verma S, Kesh K, Ganguly N, Jana S, et al. (2014). Matrix metalloproteinases and gastrointestinal cancers: Impacts of dietary antioxidants. World J. Biol. Chem. 5: 355-376.

Yedinak CG and Fleseriu M (2014). Self-perception of cognitive function among patients with active acromegaly, controlled acromegaly, and non-functional pituitary adenoma: a pilot study. Endocrine 46: 585-593. 